\title{
The Difference Between Polymyxin B and Polymyxin E in Causing Skin Hyerpigmentation
}

\author{
Dongna Zou ${ }^{1}$, Haitao $\mathrm{Yu}^{2}$ and Feifei $\mathrm{Li}^{3 *}$ \\ ${ }^{1}$ Department of Pharmacy, Shandong Provincial Hospital Affiliated to Shandong First Medical University, Jinan, China, \\ ${ }^{2}$ Department of Pharmacy, Zao Zhuang Municipal Hospital, Zao Zhuang, China, ${ }^{3}$ Department of Infection, Shandong Provincial \\ Hospital Affiliated to Shandong First Medical University, Jinan, China
}

Keywords: polymyxin B, polymyxin E, skin hyerpigmentation, histamine, melanocytes, oxidative stress, phenylalanine

OPEN ACCESS

Edited by:

Bruno Annalisa,

University of Studies G. d'Annunzio Chieti and Pescara, Italy

Reviewed by: Zhu Yan,

Monash University, Australia

Zhao Jinxin,

Monash University, Australia

*Correspondence:

Feifei $\mathrm{LI}$

slyylff@126.com

Specialty section: This article was submitted to Inflammation Pharmacology,

a section of the journal

Frontiers in Pharmacology

Received: 30 December 2020 Accepted: 24 March 2021

Published: 16 April 2021

Citation:

Zou D, YU H and Li F (2021) The Difference Between Polymyxin $B$ and

Polymyxin E in Causing

Skin Hyerpigmentation.

Front. Pharmacol. 12:647564.

doi: $10.3389 /$ fphar.2021.647564
As we all know, Coronavirus Disease 2019 spread all over the world and had became a public international event of global concern. Among Coronavirus Disease 2019 patients in China, two doctors, Yi Fan and Weifeng $\mathrm{Hu}$, were noticed with their skin pigmentary disorder due to polymyxin B. However, we found that polymyxin E has almost no reports of skin hyperpigmentation, but polymyxin B was reported about skin hyperpigmentation, although the number of relevant reports was small, what causes the difference between the polymyxin B and polymyxin $\mathrm{E}$ ?

Polymyxin is a general term for a group of basic peptide antibiotics, including A, B, C, D and E mainly. At present, the two commonly used clinically are polymyxin B and polymyxin E.

The mechanism of polymyxin B causing skin hyperpigmentation may include the following aspects:

1. Polymyxin B activates mast cells to release histamine, which binds to the $\mathrm{H}_{2}$ receptor on the surface of melanocytes inducing the stimulation of melanin synthesis

Polymyxin B is a mast cell activator that binds to non-selective $G$ protein-coupled receptors on the surface of mast cells, activates subsequent intracellular signaling pathways, and induces mast cells to degranulate and release histamine (Bushby and Green, 1955; Ferry et al., 2002; Morrison et al., 1974; Morrison et al., 1978; Zhan et al., 2019). Histamine is an inflammatory mediator involved in stimulating melanin production. Histamine binds to the $\mathrm{H}_{2}$ receptor on the surface of melanocytes in the basal layer to induce the production of cyclic adenosine monophosphate and the activation of protein kinase a in melanocytes, which leads to phosphorylation of members of the cyclic adenylate response element binding protein transcription factor family. Cyclic AMP response element binding protein activates a variety of genes and induces the transcription of a variety of enzymes and proteins related to melanin synthesis. Eventually leads to increased melanin synthesis in the cytoplasm (Yoshida et al., 2020).

Polymyxin E can also release histamine equivalent to polymyxin B (Bushby and Green, 1955), the reason why polymyxin $\mathrm{E}$ does not lead to skin hyperpigmentation is not very clear, the difference of amino acid at position 6 between the two drugs and the lower conversion rate of polymyxin $\mathrm{E}$ in vivo are possible explanation. According to the research, colistin methanesulfonate (CMS) as the prodrug is predominantly cleared by renal excretion, with only a relatively small fraction of the dosage converted to the active antibacterial in renally healthy individuals (Li et al., 2004; Li et al., 2006), it is evident that in patients with moderate to good renal function, administration of a daily dose of colistin base activity (CBA) at the upper limit of the current product-recommended dose range (300 mg CBA per day) was not able to generate plasma colistin concentrations that would be expected to be reliably efficacious (Garonzik et al., 2011). Is the concentration of polymyxin E not 
enough to activate mast cells to release enough histamine for inducing the stimulation of melanin synthesis? Further study is worthy.

2. The skin inflammation process is related to the activation of melanocytes

Histological and immunohistochemical results of pigmented skin in patients with polymyxin B

treatment showed an abundant melanocyte-pigmented dendritic network. Langerhans cells' hyperplasia and dermal IL-6 overexpression were also found, presumably for an inflammatory process due to polymyxin B use (Mattos et al., 2016; Li et al., 2020; Wen et al., 2020); At the same time, although hyperpigmented skin did not show signs of inflammationby clinical inspection, under microscopic view the dermis contained mild-to- moderate perivascular inflammatory infiltrate of lymphocytes and histiocytes. Langerhans cells are antigen-presenting cells, which play an important role in skin immunity and inflammation. The proliferation of Langerhans cells in the epidermis of patients with polymyxin B inducedpigmentation indicates that polymyxin $\mathrm{B}$ can induce the inflammatory process of the skin. In addition to its known inflammatory effects, IL-6 also inhibits the proliferation and melanogenesis of human melanocytes, When the skin is hyperpigmented, IL-6 is often feedback overexpression which may be for regulation (Jawdat et al., 2004; Mattos et al., 2017). At the same time, studies have shown that mast cell-derived factors (including histamine) can stimulate Langerhans cell migration and are related to the melanin production pathway (Miori et al., 1990).

Matzneller et al. reported that polymyxin E could decrease inflammatory cytokines, including IL-6, in the blood of $\mathrm{L}$ lipopolysaccharide-challenged healthy volunteers in a model of human endotoxiemia (Matzneller et al., 2017). And according to the newest report, it was showed that polymyxin E can't regulate the expression of the inflammatory cytokine IL6, IL6 mRNA expression levels were not changed after administration of polymyxin E (Ubagai et al., 2021).

According to these findings, the effects of polymyxin B and polymyxin E on IL6 are different. We speculate that it may explain the difference in pigmentation between the two drugs.

3. Oxidative stress is also considered to be one of the mechanisms of pigmentation (Zavascki et al., 2016)

Ahmed et al. (2017) studied the effect of polymyxin B on human lung epithelial cells A549, and the results showed that polymyxin B induced oxidative stress and loss of mitochondrial membrane potential. Compared to untreated control cells, after $8 \mathrm{~h}$ treatment, the cellular oxidative stress increased around 1.9-fold and 3.8-fold for 1.0 and $2.0 \mathrm{mM}$ of polymyxin $\mathrm{B}$, respectively, which increased up to 2.6 -fold and 4.7 -fold at $24 \mathrm{~h}$ correspondingly. The article also mentioned that the hydrophobicity of the 6phenylalanine in the structure of polymyxin B and the cationic form under physiological conditions play a key role in cytotoxicity and mitochondrial oxidative stress. This may explain the reason that why the pigmentation had not been found in the patient who used polymyxin E. In the results of polymyxin B nephrotoxicity studies, it was found that mitochondrial stress response and the production of reactive oxygen species were found in renal tubular cells treated with polymyxin B (Azad et al., 2015). Reactive oxygen species such as NO can induce the activation of guanylate cyclase and enhance the expression of tyrosinase gene to increase melanin production (Sasaki et al., 2000).

\section{Phenylalanine increases melanin synthesis (Martindale, 2014)}

Compared with polymyxin E, polymyxin B has a different structure at position 6 . Polymyxin $B$ is phenylalanine at position 6 , and polymyxin E is leucine. According to Martindale records, vitiligo can be treated with phenylalanine, and more than $60 \%$ of patients have skin pigmentation during the treatment process (Kopple et al., 2007). This could be explained by that phenylalanine is hydroxylated in the body to form tyrosine (Chang et al., 2009), which is a non-essential amino acid in the human body and the main raw material for the synthesis of melanin (Rzepka et al., 2016). And we have not seen any reports of leucine-induced skin hyperpigmentation, that maybe another reason why polymyxin E-related pigmentation has not been reported.

The nephrotoxicity and neurotoxicity induced by polymyxin B have been proved to be dose-dependent (John et al., 2017), but there is no direct evidence that polymyxin B-induced skin hyerpigmentation is a dose-dependent adverse reaction. But it was speculated that acute kidney injury (AKI) with lower creatinine clearance may be an important factor for polymyxin B-induced pigmentary disorder (Zheng et al., 2018; Lu and Hou, 2020). In addition, neonates and infants were more likely to suffered from skin hyerpigmentation after polymyxin B administration than adults, it was reported that 16 infants in ICU generalized skin hyperpigmentation in premature infants receiving polymyxin B (Shih and Gaik, 2014), and it was also noted generalized skin hyperpigmentation among neonates receiving IV polymyxin B (Gothwal et al., 2016), according to these findings, we speculate that it may be related to their immature kidney function leading to the cumulation of polymyxin B (Gothwal et al., 2016; Li et al., 2020), as polymyxin $\mathrm{B}$ is excreted through the kidney, while the incidence of skin hyperpigmentation were $15 \%$ or $8 \%$ of adult patients (Mattos et al., 2016; Mattos et al., 2017).

It should be pointed out that there have been no reports of inhalation of polymyxin B-induced skin hyperpigmentation so far, the administration for all patients suffered from skin hyerpigmentation induced by polymyxin B were intravenous (Knueppel and Rahimian, 2007; Shih and Gaik, 2014; Zavascki et al., 2015; Gothwal et al., 2016; Mattos et al., 2016; Zavascki et al., 2016; Lahiry et al., 2017; Mattos et al., 2017; Zheng et al., 2018), which is worthy of further discussion on the relationship between the administration and adverse drug reaction.

According to the findings above, we speculate boldly that polymyxin B needs to reach a certain concentration in blood to cause pigmentation and that reducing the dosage may be an effective way to prevent skin hyperpigmentation, however, 
reducing the dosage of polymyxin $\mathrm{B}$ may be likely to reduce the efficacy and even lead to bacterial resistance. It should be point out that there is no definite evidence that the occurrence of pigmentation is related to the increased concentration of polymyxin B in blood, and there is no report of dosage adjustment after the occurrence of skin pigmentation.

Regarding the difference between polymyxin $\mathrm{B}$ and polymyxin $\mathrm{E}$ in causing skin pigmentation, further research is needed in the future, and further research is needed on how to prevent and treat polymyxin B-induced skin pigmentation. However, the current related reports can remind us that we should pay attention to monitoring related adverse reactions

\section{REFERENCES}

Ahmed, M. U., Velkov, T., Lin, Y.-W., Yun, B., Nowell, C. J., Zhou, F., et al. Potential toxicity of polymyxins in human lung epithelial cells. Antimicrob. Agents Chemother. 2017;61(6):e02690-16. Published 2017 May 24. doi:10.1128/ AAC.02690-16

Azad, M. A. K., Akter, J., Rogers, K. L., Nation, R. L., Velkov, T., and Li, J. Major pathways of polymyxin-induced apoptosis in rat kidney proximal tubular cells. Antimicrob. Agents Chemother. 2015;59(4):2136-2143. doi:10.1128/AAC. 04869-14

Bushby, S. R. M., and Green, A. F. The release of histamine by polymyxin B and polymyxin E. Br. J. Pharmacol. Chemother. 1955;10(2):215-219. doi:10.1111/j. 1476-5381.1955.tb00085.x

Chang, T.-S. An updated review of tyrosinase inhibitors. Ijms. 2009;10(6): 2440-2475. Published 2009 May 26. doi:10.3390/ijms10062440

Ferry, X., Brehin, S., Kamel, R., and Landry, Y. G protein-dependent activation of mast cell by peptides and basic secretagogues. Peptides. 2002;23(8):1507-1515. doi:10.1016/s0196-9781(02)00090-6

Garonzik, S. M., Li, J., Thamlikitkul, V., Paterson, D. L., Shoham, S., Jacob, J., et al. Population pharmacokinetics of colistin methanesulfonate and formed colistin in critically ill patients from a multicenter study provide dosing suggestions for various categories of patients. Antimicrob. Agents Chemother. 2011;55(7): 3284-3294. doi:10.1128/AAC.01733-10

Gothwal, S., Meena, K., and Sharma, S. D. (2016). Polymyxin B induced generalized hyperpigmentation in neonates. Indian J. Pediatr. 83, 179-180. doi:10.1007/ s12098-015-1798-z

Jawdat, D. M., Albert, E. J., Rowden, G., Haidl, I. D., and Marshall, J. S. IgEmediated mast cell activation induces Langerhans cell migration in vivo. J. Immunol. 2004;173(8):5275-5282. doi:10.4049/jimmunol.173.8.5275

John, J. F., Falci, D. R., Rigatto, M. H., Oliveira, R. D., Kremer, T. G., and Zavascki, A. P. Severe infusion-related adverse events and renal failure in patients receiving high-dose intravenous polymyxin B. Antimicrob. Agents Chemother. 2017;62(1):e01617-17. Published 2017 Dec 21. doi:10.1128/AAC. 01617-17

Knueppel, R. C., and Rahimian, J. Diffuse cutaneous hyperpigmentation due to tigecycline or polymyxin B. Clin. Infect. Dis. 2007, 45(1): 136. doi:10.1086/ 518706

Kopple, J. D. Phenylalanine and tyrosine metabolism in chronic kidney failure. J. Nutr. 2007;137(6 Suppl. 1):1586S-1590S. doi:10.1093/jn/137.6.1586S

Lahiry, S., Choudhury, S., Mukherjee, A., Bhunya, P. K., and Bala, M. Polymyxin B-induced diffuse cutaneous hyperpigmentation. J. Clin. Diagn. Res. 2017, 11(2): FD01. doi:10.7860/JCDR/2017/24278.9213

Li, J., Milne, R. W., Nation, R. L., Turnidge, J. D., Smeaton, T. C., and Coulthard, K. Pharmacokinetics of colistin methanesulphonate and colistin in rats following an intravenous dose of colistin methanesulphonate. J. Antimicrob. Chemother. 2004;53(5):837-840. doi:10.1093/jac/dkh167

Li, J., Nation, R. L., Turnidge, J. D., Milne, R. W., Coulthard, K., Rayner, C. R., et al. Colistin: the re-emerging antibiotic for multidrug-resistant Gram-negative bacterial infections. Lancet Infect. Dis. 2006;6(9):589-601. doi:10.1016/ S1473-3099(06)70580-1 when applying polymyxin B. If skin hyerpigmentation occurs, provide corresponding psychological counseling to the patient, or take corresponding treatment measures such as laser cosmetic therapy and topical whitening agents, or adjust the dosing regimen if necessary.

\section{AUTHOR CONTRIBUTIONS}

FL was responsible for the study conception and design. DZ drafted the manuscript. HY Searched the literature. All authors contributed to the article and approved the submitted version.

Li, Y. M., Milikowski, C., Selvaggi, G., Abbo, L. M., Skiada, D., and Galimberti, F. Polymyxin B-induced skin hyperpigmentation. Transpl. Infect. Dis. 2020;22(5): e13312. doi:10.1111/tid.13312

Lu, C., and Hou, N. (2020) Skin hyperpigmentation in Coronavirus Disease 2019 patients: is polymyxin B the culprit? Front. Pharmacol. 11:01304. doi:10.3389/ fphar.2020.01304

Martindale (2014):The complete drug reference[M].38 th ed,London, United Kingdom,Pharmaceutical Press:2086

Mattos, K. P. H., Cintra, M. L., Gouvêa, I. R., Ferreira, L. Á., Velho, P. E. N. F., and Moriel, P. Skin hyperpigmentation following intravenous polymyxin B treatment associated with melanocyte activation and inflammatory process. J. Clin. Pharm. Ther. 2017;42(5):573-578. doi:10.1111/jcpt.12543

Mattos, K. P. H., Lloret, G. R., Cintra, M. L., Gouvêa, I. R., Betoni, T. R., Mazzola, P. G., et al. Acquired skin hyperpigmentation following intravenous polymyxin $\mathrm{B}$ treatment: a cohort study. Pigment Cel Melanoma Res. 2016;29(3):388-390. doi:10.1111/pcmr.12468

Matzneller, P., Strommer, S., Drucker, C., Petroczi, K., Schörgenhofer, C., Lackner, E., et al. Colistin reduces LPS-triggered inflammation in a human sepsis ModelIn vivo:A randomized controlled trial. Clin. Pharmacol. Ther. 2017; 101(6):773-781. doi:10.1002/cpt.582

Miori, L., Vignini, M., and Rabbiosi, G. Flagellate dermatitis after bleomycin. The Am. J. Dermatopathology. 1990;12(6):598-602. doi:10.1097/00000372199012000-00011

Morrison, D. C., Roser, J. F., Henson, P. M., and Cochrane, C. G. Activation of rat mast cells by low molecular weight stimuli. J. Immunol. 1974;112(2):573-582. doi:10.1115/PVP2004-2756

Morrison, D. C., Roser, J. F., Curry, B. J., Henson, P. M., and Ulevitch, R. J. Two distinct mechanisms for the initiation of mast cell degranulation. Inflammation. 1978;3(1):7-25. doi:10.1007/BF00917318

Rzepka, Z., Buszman, E., Beberok, A., and Wrześniok, D. From tyrosine to melanin: signaling pathways and factors regulating melanogenesis. Postepy Hig Med. Dosw 2016;70(0):695-708. Published 2016 Jun 30. doi:10.5604/17322693. 1208033

Sasaki, M., Horikoshi, T., Uchiwa, H., and Miyachi, Y. Up-regulation of tyrosinase gene by nitric oxide in human melanocytes. Pigment Cel Res. 2000;13(4): 248-252. doi:10.1034/j.1600-0749.2000.130406.x

Shih, L. K., and Gaik, C. L. Polymyxin B induced generalized skin hyperpigmentation in infants. Jps. 2014, 6: e215. doi:10.17334/jps.10375

Ubagai, T., Sato, Y., Kamoshida, G., Unno, Y., and Ono, Y. Immunomodulatory gene expression analysis in LPS-stimulated human polymorphonuclear leukocytes treated with antibiotics commonly used for multidrug-resistant strains. Mol. Immunol. 2021;129:39-44. doi:10.1016/j.molimm.2020.11.012

Wen, X., Luo, C., and Lyu, W. Polymyxin B-induced skin hyperpigmentation. Case Rep. Med. 2020; 2020:1 Published 2020 Sep 18. doi:10.1155/2020/6461329

Yoshida, M., Takahashi, Y., and Inoue, S. Histamine induces melanogenesis and morphologic changes by protein kinase A activation via $\mathrm{H} 2$ receptors in human normal melanocytes. J. Invest. Dermatol. 2000;114(2):334-342. doi:10.1046/j. 1523-1747.2000.00874.x

Zavascki, A. P., Manfro, R. C., Maciel, R. A., and Falci, D. R. Head and neck hyperpigmentation probably associated with polymyxin B therapy. Ann. Pharmacother. 2015, 49(10): 1171. doi:10.1177/1060028015595643 
Zavascki, A. P., Schuster, L. F., and Duquia, R. P. Histopathological findings of pigmented lesion and recovery of natural skin colour in a patient with polymyxin B-associated diffuse hyperpigmentation. Int. J. Antimicrob. Agents. 2016;48(5):579-580. doi:10.1016/j.ijantimicag.2016.08.010

Zhan, Y., Ma, N., Liu, R., Wang, N., Zhang, T., and He, L. Polymyxin B and polymyxin $\mathrm{E}$ induce anaphylactoid response through mediation of Mas-related G protein-coupled receptor X2. Chemico-Biological Interactions. 2019;308: 304-311. doi:10.1016/j.cbi.2019.05.014

Zheng, G., Cao, L., Che, Z., Mao, E., Chen, E., and He, J. Polymyxin B-induced skin hyperpigmentation: a rare case report and literature review. BMC Pharmacol. Toxicol. 2018; 19(1):41. Published 2018 Jul 4. doi:10.1186/s40360-018-0226-1
Conflict of Interest: The authors declare that the research was conducted in the absence of any commercial or financial relationships that could be construed as a potential conflict of interest.

Copyright () $2021 \mathrm{Zou}, \mathrm{Yu}$ and Li. This is an open-access article distributed under the terms of the Creative Commons Attribution License (CC BY). The use, distribution or reproduction in other forums is permitted, provided the original author(s) and the copyright owner(s) are credited and that the original publication in this journal is cited, in accordance with accepted academic practice. No use, distribution or reproduction is permitted which does not comply with these terms. 